G De Salvo' ${ }^{1}$ SR Hannan ${ }^{1}$, N James ${ }^{1}$ and AJ Lotery ${ }^{2}$

\section{Retinal angiomatous
proliferation occurring \\ Retinal angiomatous
proliferation occurring after radiotherapy}

\begin{abstract}
Purpose To describe two cases of retinal angiomatous proliferation (RAP)-like lesion following radiation therapy for primary tumor. Patients and methods Retrospective evaluation of two patients with previous irradiation treatment for a pleomorphic adenoma of the lacrimal gland and a vocal cord carcinoma, respectively. Visual acuity (VA), fluorescein angiography and optical coherence tomography were performed and demonstrated a RAP-like lesion in both cases. Treatment with intravitreal injections of Ranibizumab was performed with a follow-up of 19 and 10 months, respectively. Results Both the patients had a positive response to the treatment with improvement in VA and reduction of intraretinal fluid. Conclusion RAP-like lesions can develop following radiation treatment for a primary tumor. In patients presenting with idiopathic RAP, a history of prior radiotherapy should be considered.

Eye (2013) 27, 447-449; doi:10.1038/eye.2012.274; published online 4 January 2013
\end{abstract}

Keywords: retinal angiomatous proliferation; Type 3 neovascularization; radiation retinopathy; ranibizumab

\section{Introduction}

Retinal angiomatous proliferation (RAP) is a subtype of age-related macular degeneration described by Yannuzzi et al ${ }^{1}$ in 2000. More recently the term 'Type 3 neovascularization'2,3 has been adopted as a synonym for RAP. We describe two cases of RAP-like lesion secondary to radiation treatment for primary tumor.

\section{Case reports}

\section{Case 1}

An 83-year-old woman was referred to the retina clinic because of distortions in OD.
In 2003 she underwent surgical excision and radiation therapy for a pleomorphic adenoma of the right lacrimal gland. A month after the radiotherapy she developed a right retinal detachment, which underwent vitrectomy with good visual acuity (VA). She also underwent surgery for a right cataract and right ptosis. At presentation VA was $0.91 \log$ MAR OD and $0.12 \log$ MAR OS. Ophthalmic examination demonstrated right mild ptosis and superficial punctate keratitis. Fundus examination showed intraretinal exudates, intraretinal hemorrhages, and an elevated macula (Figure 1a). Fluorescein angiography (FA) demonstrated early hyperfluorescence due to intraretinal neovascularization (IRN) indicating RAP (Figure 1b). Optical coherence tomography (OCT) was also performed through the fovea and confirmed the IRN finding together with intraretinal fluid (IRF) (Figure 1c). OS was unremarkable. A diagnosis of RAP-like lesion was made and she was started on a loading dose of three intravitreal injections of Ranibizumab, given monthly followed by treatment pro re nata (prn). Retreatment criteria were based on the OCT appearance showing persistent or new IRF. The patient has a follow-up of 19 months during, which she received 14 Ranibizumab injections. VA improved to $0.62 \log$ MAR and the OCT showed resolution of the INR and the IRF (Figure 1d).

\section{Case 2}

An 82-year-old man presented at our clinic because of a scotoma OD. He had previous bilateral cataract surgery, bilateral mild nonproliferative diabetic retinopathy (MNPDR) with right clinically significant macular edema laser treated, and epiretinal membrane OS. Seven months earlier he underwent radiotherapy for a right vocal cord carcinoma. His VA was $1.17 \log$ MAR OD and $0.36 \log$ MAR
${ }^{1}$ Southampton Eye Unit, University Hospital Southampton, Southampton, UK

${ }^{2}$ Clinical Neurosciences Research Group, Faculty of Medicine, University of Southampton, Southampton, UK

Correspondence: AJ Lotery, Southampton Eye Unit, Southampton General Hospital, Tremona Road, Southampton SO16 6YD, UK.

Tel: +44 2380794590 ;

Fax +44 2380794120 .

E-mail: A.J.Lotery@

soton.ac.uk

Received: 20 August 2012 Accepted in revised form: 18 November 2012 Published online: 4 January 2013 

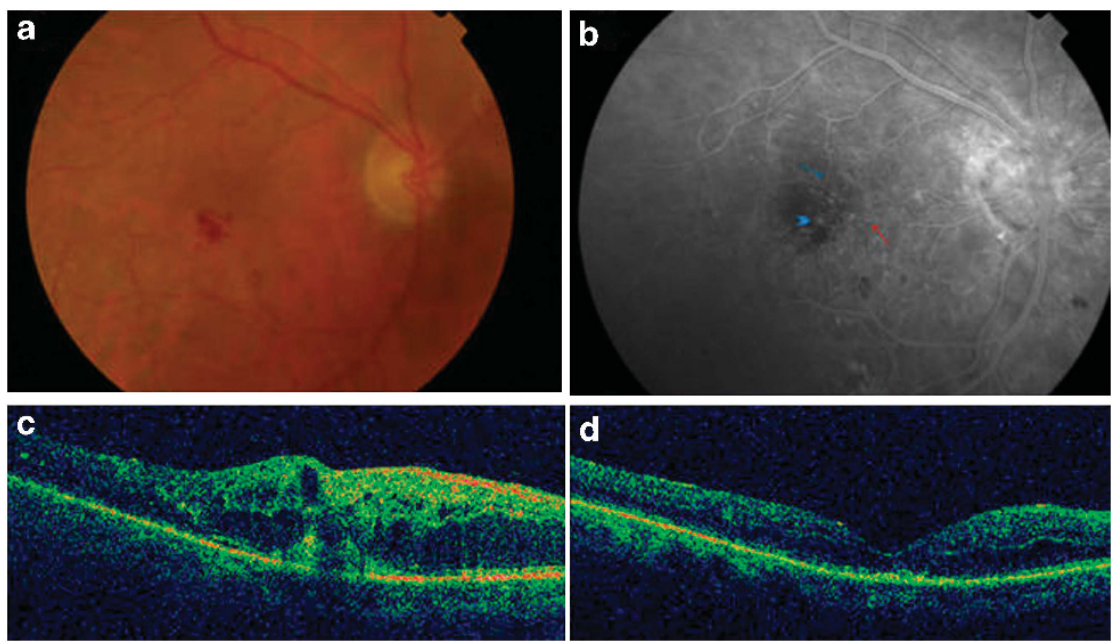

Figure 1 (a) Fundus photo OD: intaretinal exudates, intraretinal hemorrhages and an elevated macula. (b) FA: (arrow head) hyperfluorescent IRN with feeding arteriole (red arrow) and draining retinal vein (blue arrow) indicating RAP. (c) Optical coherence OCT: hyperreflectivity within the outer retinal layer indicating IRN; increased macular thickness and hyporeflectivity due to IRF; (d) OCT: resolution of the IRN and the IRF.
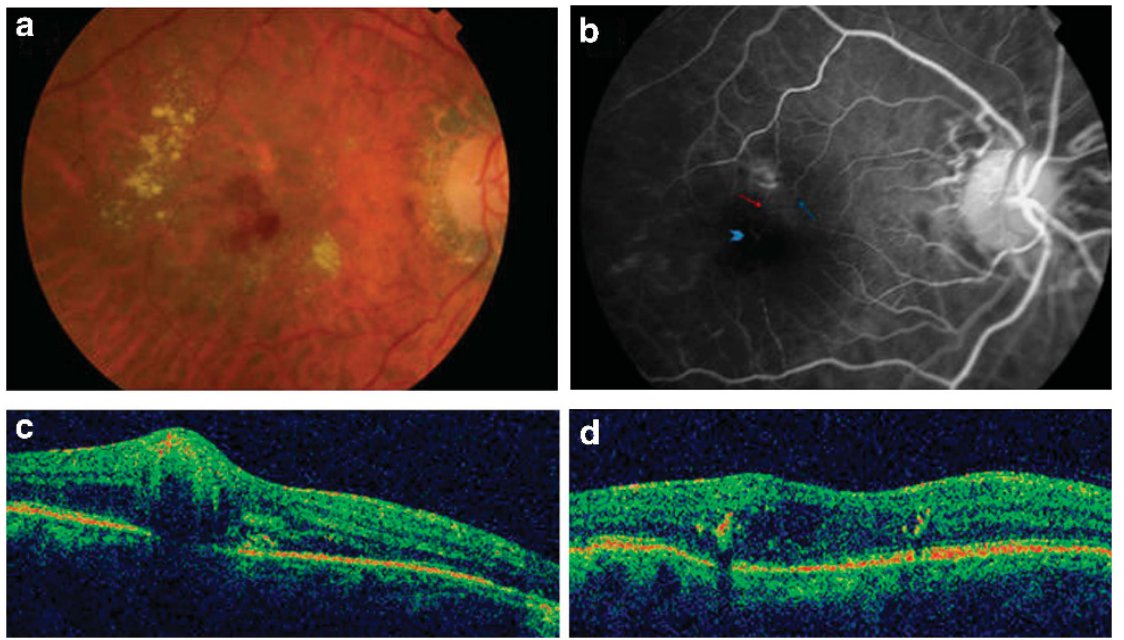

Figure 2 (a) Fundus photo OD: hard exudates, superficial and intraretinal hemorrhages. (b) FA: early hyperfluorescent anastomosis (arrow head) with feeding arteriole (red arrow) and draining retinal vein (blue arrow) (c) OCT: small pigment epithelial detachment together with IRN, IRF and subretinal fluid; (d) OCT: residual IRF.

OS. Retinal examination OD revealed hard exudates, superficial and intraretinal hemorrhages, and IRF (Figure 2a). FA highlighted an early anastomosis (Figure 2b). OCT at the fovea confirmed IRF and subretinal fluid associated with a small pigment epithelial detachment (Figure 2c). A diagnosis of a RAPlike lesion was made and he was started on a loading dose of three intravitreal Ranibizumab injections. He received six injections during 10 months follow-up. The retreatment criteria were the same as for case 1 . VA improved to $0.80 \log$ MAR but with some residual IRF (Figure 2d).

\section{Discussion}

Ophthalmic complications of radiotherapy following the treatment for a primary tumor can affect any part of the eye and its adnexa, and have been reported widely. ${ }^{4-8}$ The clinical and diagnostic findings of our patients were consistent with a RAP lesion. Although our first patient developed ophthalmic complications following irradiation, they started a year after the treatment and it was only at a later stage that she developed a RAP-like lesion. It is possible that the previous vitrectomy might have a role in delaying the development of radiation 
retinopathy. Our second case simply presented with a RAP-like lesion. In this latter case the neovascularization developed earlier, only 7 months post-treatment. Possibly his MNPDR facilitated the development of the RAP-like lesion. This patient also had laser scars owing to previous grid laser therapy, but none were located at the fovea. Furthermore, the fluorescein angiogram did not show evidence of classic choroidal neovascularization.

A previous case report ${ }^{9}$ demonstrated the development of a RAP-like lesion secondary to irradiation with different histological characteristics from a typical RAP.

Alternatively, it is also possible that the cases we report are coincidental to the previous radiotherapy and merely represent age-related macular degeneration. However, the previous history of radiotherapy and the fact that the RAP occurred on the same side of the treatment, together with the lack of drusen and RPE changes in the other eye, supports the hypothesis that these two patients developed a RAP-like lesion secondary to radiation.

Strenja-Linić et $a l^{10}$ found microangiopathic changes after radiotherapy for laryngeal carcinoma only in those patients with increased cerebrovascular risks such as diabetes mellitus and arterial hypertension. This would explain why our second case developed a RAP-like lesion relatively earlier compared with our first case, which did not have any cerebrovascular risks. In both of our patients with previous history of radiotherapy, the fact that the RAP occurred on the same side of the treatment and the lack of involvement of the contralateral eye would support the hypothesis that their RAP-like lesion is secondary to the radiation. Also, an association between previous radiation exposure and idiopathic perifoveal telangiectasis ${ }^{11}$ has been proved. 'The proliferative vasogenetic sequence in RAP is very similar to IPT', but without a choroidal component. $^{3}$

In conclusion, we believe that RAP should be considered as one of the possible complications of radiation treatment for a primary tumor of the head and the neck. A history of previous radiotheraphy should be queried in patients presenting with RAP. Further research is required in order to examine the possibility of radiation having a role in the pathophysiology of RAP.
Summary

What was known before

- Radiation retinopathy and choroidal neovascularization are well-known complications of radiation therapy of the neck and head.

What this study adds

- These two cases are showing two RAP-like lesions, which developed following radiation treatment of the head and neck in two different patients. This is a new association.

\section{Conflict of interest}

The authors declare no conflict of interest.

\section{References}

1 Yannuzzi LA, Negrao S, Iida T, Carvalho C, RodriguezColeman $\mathrm{H}$, Slakter $\mathrm{J}$ et al. Retinal angiomatous proliferation in age-related macular degeneration. Retina 2001; 21: 416-434.

2 Gass JD. Stereoscopic Atlas of Macular Diseases. 4th edn. Mosby: Maryland Heights, USA, 1997, pp 26-30.

3 Yannuzzi LA, Freund KB, Takahashi BS. Review of retinal angiomatous proliferation or type 3 neovascularization. Retina 2008; 28(3): 375-384.

4 Parsons JT, Bova FJ, Mendenhall WM, Million RR, Fitzgerald CR. Response of the normal eye to high dose radiotherapy. Oncology 1996; 10(6): 837-847.

5 Archer DB, Amoaku WM, Gardiner TA. Radiation retinopathy - clinical, histopathological, ultrastructural and experimental correlations. Eye 1991; 5(Part 2): 239-251.

6 Boozalis GT, Schachat AP, Green WR. Subretinal neovascularization from the retina in radiation retinopathy. Retina 1987; 7: 156-161.

7 Spaide RF, Borodoker N, Shah V. Atypical choroidal neovascularization in radiation retinopathy. $A m \mathrm{~J}$ Ophthalmol 2002; 133(5): 709-711.

8 Berker N, Aslan O, Batman C, Elgin U, Ozkan SS. Choroidal neovascular membrane in radiation retinopathy. Clin Experiment Ophthalmol 2006; 34(6): 625-626.

9 Anzai K, Mori K, Yoneya S. Clinicopathological correlation of a retinal angiomatous proliferation-like lesion in a case of radiation retinopathy. Jpn J Ophthalmol 2006; 50(3): 291-293.

10 Strenja-Linić I, Vojniković B, Caljkusić-Mance T, Tićac R, Bonifacić D, Kovacević D. Focused high risk-population screening for carotid stenosis and retinal microangiopathia after radiotherapy for laryngeal carcinoma. Coll Antropol 2010; 34(Suppl 2): 49-52.

11 Maberley DA, Yannuzzi LA, Gitter K, Singerman L, Chew E, Freund $\mathrm{KB}$ et al. Radiation exposure: a new risk factor for idiopathic perifoveal telangiectasis. Ophthalmology 1999; 106(12): 2248-2252. 